\title{
AUTHOR INDEX (Volume 16)
}

\section{A}

Agarwal, M. \& Sharma, V. K., Ant Colony Optimization Algorithm for Heterogeneous Redundancy Allocation in Multi-State Series-Parallel Systems

Aggarwal, A. G., see Kapur, P. K.

Aggarwal, A. G., see Kapur, P. K.

Aronov, J., see Papic, L.

B

Beaujean, P.-P., see Sloan, J. C.

Bes, C., see Saintis, L.

Bhattacharjya, S. \& Chakraborty, S., Robust Optimization of Linear Dynamic System with Random Parameters Under Stochastic Earthquake Excitation

Bullard, L. A., see Khoshgoftaar, T. M.

C

Castagliola, P., Celano, G. \& Chen, G., The Exact Run Length Distribution and Design of the $S^{2}$ Chart When the In-control Variance Is Estimated

1 (2009) 23

1 (2009) 23

Celano, G., see Castagliola, P.

Chakraborty, S., see Bhattacharjya, S.

3 (2009) 261

1 (2009) 23

Chen, Z. \& Ye, C., An Alternative Test for Uniformity

4 (2009) 343

D

Driscoll, F., see Sloan, J. C.

5 (2009) 413

F

Fiondella, L., Statistical Inference and Uncertainty Assessment for Multi-State Systems

5 (2009) 453

Fontaine, J. G., see Mennis, E. 1 (2009) 59
G

Gao, K., see Khoshgoftaar, T. M.

1 (2009) 73

Garg, R. B., see Kapur, P. K.

6 (2009) 567

H

Hsieh, Y.-W., see Tsai, T.-R. 1 (2009) 1

Hugues, E., see Saintis, L.

2 (2009) 91

I

Inoue, S., Iwamoto, N. \& Yamada, S., Debugging Process-Oriented Discrete Software Reliability Modeling

4 (2009) 357

Iwamoto, N., see Inoue, S. $\quad 4$ (2009) 357

K

Kapoor, K., see Kapur, P. K.

Kapur, P. K., Aggarwal, A. G., Kapoor, K. \& Kaur, G., Optimal Testing Resource Allocation for Modular Software Considering Cost, Testing Effort and Reliability Using Genetic Algorithm

6 (2009) 495

Kapur, P. K., Garg, R. B., Aggarwal, A. G. \& Tandon, A., General Framework for Change Point Problem in Software Reliability and Related Release Time Problem

Karki, N. R., Verma, A. K., Karki, R. \& Mishra, A. K., Residential Customers' Outage Cost Analysis for Urban and Semi-urban Areas in a Developing Country

6 (2009) 495

6 (2009) 567

Karki, R., see Karki, N. R.

Kaur, G., see Kapur, P. K.

6 (2009) 581

6 (2009) 581

6 (2009) 495

5 (2009) 435

Kawahara, A., see Yamada, S.

Khoshgoftaar, T. M., see Sloan, J. C.

2 (2009) 137

Khoshgoftaar, T. M., see Sloan, J. C.

5 (2009) 413

Khoshgoftaar, T. M., Bullard, L. A. \& Gao, K., Attribute Selection Using Rough 
Sets in Software Quality Classification

1 (2009) 73

5 (2009) 385

Kodera, T., see Tokuno, K.

L

Liao, G.-L. \& Shaw, B.-L., Optimal Maintenance Policy to Support the Operation of Manufacturing with Minimal and Delayed Repair

Lundteigen, M. A. \& Rausand, M., Reliability Assessment of Safety Instrumented Systems in the Oil and Gas Industry: A Practical Approach and a Case Study

\section{M}

Manjunatha, P., Verma, A. K. \& Srividya, A., Routing Path Optimizaion to Maximize the Lifetime of Wireless Sensor Network

Mennis, E., Platis, A., Nikitakos, N. \& Fontaine, J. G., Enhancing Safety in Ship's Critical Systems Using Markov Modeling

Miladinovic, B. \& Tsokos, C. P., Sensitivity of the Bayesian Reliability Estimates for the Modified Gumbel Failure Model

Miman, M. \& Pohl, E. A., Uncertainty Assessment Techniques for System Availability

3 (2009) 235

2 (2009) 187

6 (2009) 509

1 (2009) 59

4 (2009) 331

Mishra, A. K., see Karki, N. R.

Mohan, K. K., Verma, A. K. \& Srividya, A., Early Qualitative Software Reliability Prediction and Risk Management in Process Centric Development through a Soft Computing Technique

6 (2009) 521

Mongeau, M., see Saintis, L.

2 (2009) 91

$\mathbf{N}$

Nikitakos, N., see Mennis, E. Noguchi, H., see Otsuka, Y.

1 (2009) 59

3 (2009) 281

O

Otsuka, Y., Shimizu, H. \& Noguchi, H., Efficacious Design Review Process

1 (2009) 39

6 (2009) 581
Including On-the-Job Training in Finding Misunderstand Errors

3 (2009) 281

$\mathbf{P}$

Pantelic, M., see Papic, L.

Papic, L., Aronov, J. \& Pantelic, M., Safety Based Maintenance Concept

Patel, M. H. \& Tsao, H.-S. J., Forward Apportionment of Censored Counts for Discrete Nonparametric Maximum Likelihood Estimation of Failure Probabilities

3 (2009) 213

Platis, A., see Mennis, E.

1 (2009) 59

Pohl, E. A., see Miman, M.

1 (2009) 39

$\mathbf{R}$

Rausand, M., see Lundteigen, M. A.

2 (2009) 187

$\mathbf{S}$

Saintis, L., Hugues, E., Bes, C. \& Mongeau, M., Computing In-Service Aircraft Reliability

Schneidewind, N., Software Risk Analysis

Sharma, V. K., see Agarwal, $M$.

6 (2009) 533

6 (2009) 533

Shaw, B.-L., see Liao, G.-L.

Shimizu, H., see Otsuka, Y.

Sloan, J. C. \& Khoshgoftaar, T. M., Testing and Formal Verification of Service Oriented Architectures

2 (2009) 137

Sloan, J. C., Khoshgoftaar, T. M., Beaujean, P.-P. \& Driscoll, F., Ocean Turbines - A Reliability Assessment

2 (2009) 91

2 (2009) 117

2 (2009) 163

3 (2009) 235

3 (2009) 281

5 (2009) 413

Srinivas, G., Verma, A. K. \& Srividya, A., Risk Informed Decision Making Using Multiobjective Optimization Technique

Srividya, A., see Manjunatha, $\mathrm{P}$.

6 (2009) 483

6 (2009) 509

6 (2009) 521

6 (2009) 483

Srividya, A., see Srinivas, G.

Stehlík, M., Scale Testing in Small Samples with Missing Time-to-Failure Information

6 (2009) 469 
$\mathbf{T}$

Tamura, Y. \& Yamada, S., An Embedded Oss Reliability and Optimization Analysis Incorporating Imperfect Debugging

Tandon, A., see Kapur, P. K.

Tokuno, K., Kodera, T. \& Yamada, S., Generalized Markovian Software Reliability Modeling and Its Alternative Calculation

Trivedi, K. S., see Wang, D.

Tsai, T.-R. \& Hsieh, Y.-W., Simulated Shewhart Control Chart for Monitoring Variance Components

Tsao, H.-S. J., see Patel, M. H.

Tsokos, C. P., see Miladinovic, B.

V

Venu, V. V. \& Verma, A. K., Reinforcement of Power System Reliability Measures through Joint Deterministic and Probabilistic Approaches

6 (2009) 551

Verma, A. K., see Karki, N. R.

Verma, A. K., see Manjunatha, $\mathrm{P}$.

4 (2009) 371

6 (2009) 567

5 (2009) 385

1 (2009) 1

3 (2009) 213

4 (2009) 331

6 (2009) 581
Verma, A. K., see Mohan, K. K.

6 (2009) 521

Verma, A. K., see Srinivas, G.

Verma, A. K., see Venu, V. V.

6 (2009) 483

6 (2009) 551

W

Wang, D. \& Trivedi, K. S., Modeling User-Perceived Reliability Based on User Behavior Graphs

4 (2009) 303

Wang, Z., see Xie, L.

3 (2009) 249

4 (2009) 303

\section{$\mathrm{X}$}

Xie, L. \& Wang, Z., Loadstrength Interference Failure Rate Model

3 (2009) 249

$\mathbf{Y}$

Yamada, S. \& Kawahara, A., Statistical Analysis of Process Monitoring Data for Software Process Improvement

5 (2009) 435

4 (2009) 357

Yamada, S., see Inoue, S.

4 (2009) 371

5 (2009) 385

Yamada, S., see Tokuno, K.

Yang, G., Design for Reliability and Robustness: A Case Study

6 (2009) $509 \quad$ Ye, C., see Chen, Z. 4 (2009) 343 